\title{
First-pass contrast bolus hemodynamics contain information on right ventricular function, remodeling, and lung resistance in pulmonary arterial hypertension patients
}

Jan Skrok ${ }^{1 *}$, Monda L Shehata ${ }^{1}$, Thomas Goldstein ${ }^{2}$, Jie Zheng ${ }^{3}$, Reda E Girgis ${ }^{1}$, James O Mudd ${ }^{1}$, Joao AC Lima', David A Bluemke ${ }^{4}$, Paul M Hassoun', Jens Vogel-Claussen'

From 2011 SCMR/Euro CMR Joint Scientific Sessions

Nice, France. 3-6 February 2011

\section{Background}

In pulmonary arterial hypertension (PAH), increased vascular resistance causes functional and structural changes in the right ventricle (RV), ultimately leading to right heart failure and death. Predictors of patient survival include RV cardiac index (RVCI) and pulmonary vascular resistance (PVR). First-pass contrast bolus hemodynamics, such as cardiopulmonary transit time (PTT), full-width-half-maximum (FWHM), and time-to-peak, have been associated with left ventricular (LV) function; however, their relation to RV function and pulmonary hemodynamics as well as their significance in PAH have not been investigated.

\section{Purpose}

To evaluate first-pass contrast bolus hemodynamic parameters in relation to biventricular function and pulmonary hemodynamics in patients undergoing right heart catheterization (RHC) for known or suspected PAH.

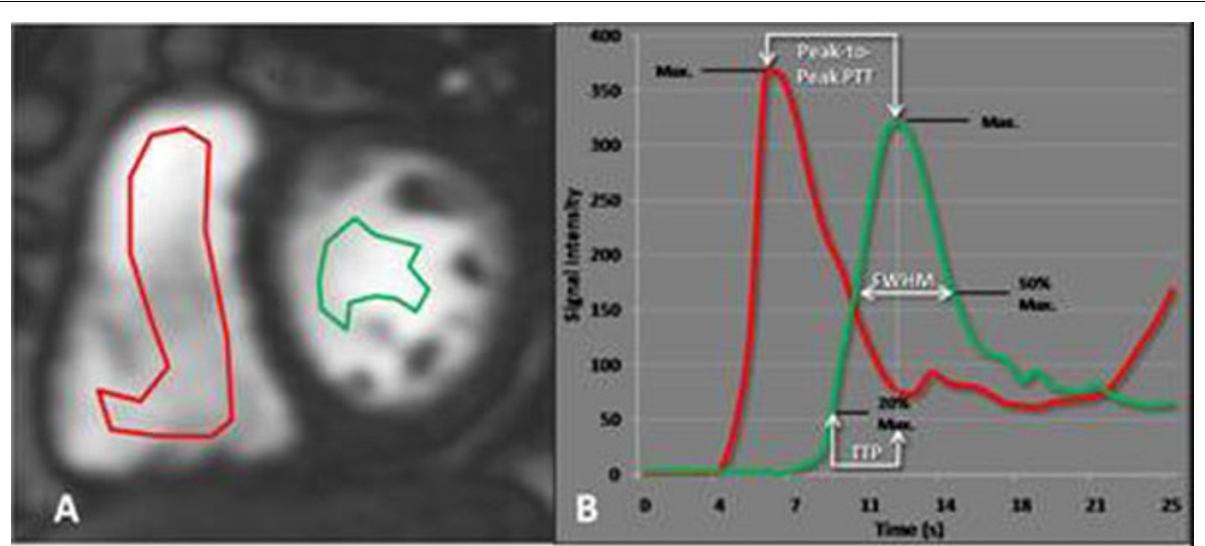

Figure $1 \mathrm{ROI}$ Placement and Time-Intensity Curves. Short-axis saturation-recovery GRE image (A) demonstrates regions of interest placed in the right (red) and left (green) ventricular cavities. Time-intensity curves (B) illustrate the passage of the contrast bolus through the regions of interest and signify the calculated parameters; peak-to-peak cardiopulmonary transit time, full-width-half-maximum (FWHM), and time-to-peak (TTP)

1Johns Hopkins University School of Medicine, Baltimore, MD, USA

Full list of author information is available at the end of the article 


\section{Methods}

43 patients (36 females, 58.7 years) underwent RHC and $3 \mathrm{~T}$ cardiac MRI on the same day. 32 were confirmed to have PAH (mPAP 40 [29-49] $\mathrm{mmHg}$ ), 11 did not have PAH (mPAP 17 [15-20] $\mathrm{mmHg}$ ). 18 age- and gendermatched healthy volunteers were included.

For evaluation of bolus hemodynamics, a 1:10 diluted contrast bolus $(0.0025 \mathrm{ml} / \mathrm{min} / \mathrm{kg})$ of gadopentetate dimeglumine was administered intravenously, and a short-axis saturation-recovery GRE slice was acquired in the proximal third of both ventricles over 40 heartbeats with one image per beat. For analysis, two regions of interest were drawn in the right and left ventricular cavities, and timeintensity curves were generated. From these curves, PTT, FWHM, and time-to-peak were calculated (Figure 1).

\section{Results}

Right-to-left-ventricular PTT, LV FWHM, and LV timeto-peak for PAH patients $(8.2 \mathrm{~s}, 8.2 \mathrm{~s}, 4.8 \mathrm{~s})$ were significantly longer than for non-PAH $(6.5 \mathrm{~s}, \mathrm{p}=0.006 ; 5.0 \mathrm{~s}$, $\mathrm{p}=0.01 ; 3.6 \mathrm{~s}, \mathrm{p}=0.01)$ and control subjects $(6.4 \mathrm{~s}$, $\mathrm{p}=0.0003 ; 5.2 \mathrm{~s}, \mathrm{p}=0.0004 ; 3.2 \mathrm{~s}, \mathrm{p}<0.0001)($ Table 1$)$. There were significant correlations of all three parameters with pulmonary hemodynamics and biventricular function and structure (Table 2). In linear regression analysis, including PVRI, ventricular mass index (VMI), and $\mathrm{CI}$ as covariates, PTT was predicted by RVCI and VMI, while FWHM and time-to-peak were predicted by PVRI. In receiver operator characteristics (ROC) analysis for transit time to distinguish between $\mathrm{PAH}$ patients with and without right heart failure (RVCI $\left.<2.2 \mathrm{~L} / \mathrm{min} / \mathrm{m}^{2}\right)$ the area under the ROC curve was 0.82 with a sensitivity of $100 \%$ and specificity of $63.6 \%$ for a threshold of 8.1 s.

\section{Conclusions}

While right-to-left-ventricular PTT in PAH patients is mainly predicted by right ventricular cardiac function and biventricular remodeling, time-to-peak and FWHM are associated with pulmonary vascular resistance. Their

Table 1 Transit times and contrast bolus dispersion

\begin{tabular}{|c|c|c|c|c|}
\hline & $\begin{array}{l}\text { PAH } \\
(n=32)\end{array}$ & $\begin{array}{l}\text { Non-PAH } \\
(n=11)\end{array}$ & $\begin{array}{l}\text { Controls } \\
(n=18)\end{array}$ & $\mathbf{P}$ \\
\hline Peak Transit Time (s) & $\begin{array}{l}8.2^{\dagger+\neq \neq} \\
{[6.9-9.9]}\end{array}$ & $\begin{array}{l}6.5^{\dagger+} \\
{[5.6-7.0]}\end{array}$ & $\begin{array}{l}6.4^{\neq \neq} \\
{[5.7-7.1]}\end{array}$ & $0.0003^{*}$ \\
\hline FWHM LV (s) & $\begin{array}{l}8.2^{\dagger+\neq \neq} \\
{[5.7-11.4]}\end{array}$ & $\begin{array}{l}5.0^{\dagger \dagger} \\
{[4.0-7.3]}\end{array}$ & $\begin{array}{l}5.2^{\ddagger \neq} \\
{[4.1-6.1]}\end{array}$ & $0.0006^{*}$ \\
\hline $\begin{array}{l}\text { Time-to-Peak } \\
\text { LV (s) }\end{array}$ & $\begin{array}{l}4.8^{\dagger+\neq \neq} \\
{[3.9-6.5]}\end{array}$ & $\begin{array}{l}3.6^{\dagger \dagger} \\
{[2.7-4.0]}\end{array}$ & $\begin{array}{l}3.2^{\neq \neq} \\
{[2.8-3.8]}\end{array}$ & $0.0001^{*}$ \\
\hline
\end{tabular}

Peak-to-peak cardiopulmonary transit time, left ventricular full-width-halfmaximum (FWHM) and time-to-peak measurements were significantly longer for PAH patients than for non-PAH patients and healthy controls. MannWhitney $U$ test was used to comparison of all three groups: ${ }^{*} \mathrm{p}<0.01$. Wilcoxon rank-sum text was used for individual group comparisons: $\mathrm{PAH}$ vs non-PAH: ${ }^{\dagger} \mathrm{p}<0.05,{ }^{++} \mathrm{p}<0.01$; PAH vs. controls: ${ }^{\ddagger} \mathrm{p}<0.05,{ }^{\ddagger \neq} \mathrm{p}<0.01$.
Table 2 Correlations of Transit Time and Dispersion Coefficients with Biventricular Function and Pulmonary Hemodynamics

\begin{tabular}{|c|c|c|c|c|c|c|}
\hline & \multicolumn{2}{|c|}{$\begin{array}{l}\text { Peak-to-Peak } \\
\text { Transit Time }\end{array}$} & \multicolumn{2}{|c|}{ LV FWHM } & \multicolumn{2}{|c|}{$\begin{array}{l}\text { LV Time-to- } \\
\text { Peak }\end{array}$} \\
\hline & $r$ & $p$ & $r$ & $\mathbf{p}$ & $r$ & $\mathbf{p}$ \\
\hline Age & 0.23 & 0.13 & 0.08 & 0.64 & 0.04 & 0.80 \\
\hline $6 \mathrm{MWD}$ & -0.35 & $0.03^{*}$ & -0.23 & 0.16 & -0.32 & $0.05^{*}$ \\
\hline \multicolumn{7}{|l|}{ RHC Parameters } \\
\hline Mean RAP & 0.20 & 0.20 & 0.19 & 0.24 & 0.22 & 0.16 \\
\hline Mean PAP & 0.55 & $0.0001^{*}$ & 0.50 & $0.0008^{*}$ & 0.47 & $0.002^{*}$ \\
\hline Systolic PAP & 0.57 & $<0.0001^{*}$ & 0.50 & $0.0008^{*}$ & 0.45 & $0.003^{*}$ \\
\hline PCWP & 0.24 & 0.13 & 0.06 & 0.70 & 0.21 & 0.18 \\
\hline PVRI & 0.64 & $<0.0001^{*}$ & 0.56 & $0.0001^{*}$ & 0.54 & $0.0002^{*}$ \\
\hline RHC RV Cardiac Index & $\begin{array}{l}-0 / \\
48\end{array}$ & $0.001^{*}$ & -0.37 & $0.02^{*}$ & -0.43 & $0.004^{*}$ \\
\hline $\begin{array}{l}\text { RHC RV Stroke Volume } \\
\text { Index }\end{array}$ & -0.55 & $0.0001^{*}$ & -0.54 & $0.0002^{*}$ & -0.52 & $0.0004^{*}$ \\
\hline LV Stroke Work Index & -0.48 & $0.001^{*}$ & -0.48 & $0.002^{*}$ & -0.44 & $0.003^{*}$ \\
\hline RV Stroke Work Index & 0.30 & $0.049^{*}$ & 0.23 & 0.16 & 0.21 & 0.19 \\
\hline
\end{tabular}

\section{Cardiac MRI}

Parameters

LV ED Volume/BSA $\quad-0.42 \quad 0.006^{*} \quad \begin{array}{lllll}-0.40 & 0.009^{*} & -0.29 & 0.06\end{array}$

$\begin{array}{lllllll}\text { LV ES Volume/BSA } & -0.23 & 0.14 & -0.14 & 0.38 & 0.04 & 0.004^{*}\end{array}$

LV Stroke Volume Index $\quad-0.47 \quad 0.001^{*} \quad \begin{array}{llllll}-0.50 & 0.0009^{*} & -0.44 & 0.004^{*}\end{array}$

LV Cardiac Index $\quad-0.58<0.0001^{*}-0.45 \quad 0.003^{*} \quad-0.38 \quad 0.01^{*}$

$\begin{array}{lllllll}\text { LV EF } & -0.16 & 0.32 & -0.31 & 0.049^{*} & -0.40 & 0.008^{*}\end{array}$

$\begin{array}{lllllll}\text { LV Mass/BSA } & -0.10 & 0.53 & -0.29 & 0.07 & -0.09 & 0.59\end{array}$

$\begin{array}{lllllll}\text { RV ED Volume/BSA } & 0.22 & 0.16 & 0.09 & 0.58 & 0.15 & 0.35\end{array}$

RV ES Volume/BSA $\quad \begin{array}{lllllll}0.37 & 0.01 * & 0.30 & 0.05 & 0.33 & 0.03 *\end{array}$

RV Stroke Volume Index $-\begin{array}{llllll}0.48 & 0.001^{*} & -0.52 & 0.0006^{*} & -0.46 & 0.002^{*}\end{array}$

RV Cardiac Index $\quad-0.59<0.0001^{*}-0.45 \quad 0.003^{*} \quad-0.42 \quad 0.006^{*}$

RV EF $\quad-0.54 \quad 0.0002^{*} \quad-0.50 \quad 0.0008^{*}-0.52 \quad 0.0004^{*}$

$\begin{array}{lllllll}\text { RV Mass/BSA } & 0.47 & 0.001^{*} & 0.31 & 0.045^{*} & 0.30 & 0.05\end{array}$

$\begin{array}{lllllll}\text { Total Biventricular Mass/ } & 0.28 & 0.06 & 0.11 & 0.51 & 0.19 & 0.24\end{array}$ BSA

predictive value regarding patient prognosis warrants further investigation.

Author details

${ }^{1}$ Johns Hopkins University School of Medicine, Baltimore, MD, USA. ${ }^{2}$ Stanford University, Stanford, CA, USA. 'Washington University School of Medicine, St. Louis, MO, USA. ${ }^{4}$ National Institutes of Health, Bethesda, MD, USA.

Published: 2 February 2011

doi:10.1186/1532-429X-13-S1-P324

Cite this article as: Skrok et al.: First-pass contrast bolus hemodynamics contain information on right ventricular function, remodeling, and lung resistance in pulmonary arterial hypertension patients. Journal of

Cardiovascular Magnetic Resonance 2011 13(Suppl 1):P324. 